\title{
On a novel method of impact by a front-end-coated bullet to evaluate the interface adhesion between film and substrate
}

\author{
Zhi-Lin $\mathrm{Wu}^{\mathrm{a}}$, Chen-Wu Wu ${ }^{\mathrm{b}, *}$, Guang-Nan Chen ${ }^{\mathrm{b}}$, Kun Zhang ${ }^{\mathrm{b}}$ \\ a School of Mechanical Engineering, Nanjing University of Science and Technology, Nanjing 210094, PR China \\ ${ }^{\mathrm{b}}$ Institute of Mechanics, Chinese Academy of Sciences, Beijing 100190, PR China
}

\section{A R T I C L E I N F O}

\section{Article history:}

Received 19 May 2009

Received in revised form 6 July 2009

Accepted 15 July 2009

\section{Keywords:}

Impact by coated bullet

Interface adhesion

Film and substrate

Theoretical description

FEM

\begin{abstract}
A B S T R A C T
A preliminary experiment was carried out to validate the feasibility of the method of impact by a front-end-coated bullet to evaluate the interface adhesion between film and substrate. The theoretical description of the initiation, propagation and evolution of the stress pulse during impact was generalized and formulized. The effects of the crucial parameters on the interface stress were further investigated with FEM. The results found the promising prospect of the application of such a method and provided useful guidance for experimental design.
\end{abstract}

(c) 2009 Elsevier B.V. All rights reserved.

\section{Introduction}

Films (coatings) with resistance to wear, corrosion and thermal degradation as well as other important functions have been applied in many fields [1]. However, their prospective applications are restricted due to premature film debonding. Numerous methods $[2,3]$ have been applied to evaluate the adhesion between the film and the substrate. The key step in these experiments is to exert simple loading directly on the interface and to separate the film from the substrate, which is hard to accomplish especially when the film is strongly bonded.

The method based on the impact by a front-end-coated bullet was devised to evaluate the adhesion between the film and substrate [4]. In the method, a front-end-coated bullet accelerated by a gas gun is used to impact the substrate of a test specimen as shown in Fig. 1. Upon impact a compressive stress pulse is initiated which propagates towards the to-be-tested film. After transmitting through the interface, part of this pulse is reflected on the free surface of the film, and then, tensile stress emerges around the interface between the film and substrate. Such a tensile stress might induce the debonding of the film in test. Assuming the acoustic impedances of both the bullet and the substrate of the specimen are identical, Wu et al. [4] described qualitatively the evolution of the stress waves under an impact. It is predicted that such an

\footnotetext{
* Corresponding author at: No. 15 Beisihuanxi Road, Beijing 100190, PR China. Tel.: +8610 82544270; fax: +861062561284.

E-mail addresses: wcw_on@yahoo.com.cn, chenwuwu@imech.ac.cn (C.-W.Wu).
}

impact method can produce a short duration compressive stress pulse as well as the method of laser spallation [5-7] but, can avoid the complicated phenomena that arise in laser spallation.

In the present work, first a preliminary experiment was carried out to validate the feasibility of this method. Later on, the theoretical description of the initiation, propagation and evolution of the stress pulse during impact was generalized and formulized. The effects of the crucial parameters on the interface stress were further investigated with FEM.

\section{Preliminary experiment}

To validate the idea of this method, a preliminary experiment is conducted by means of accelerating a front-end-coated bullet with the equipment as shown in Fig. 2(a) to impact the specimen. Materials and their mechanical parameters are listed in Table 1. The initial velocity of the bullet is $565 \mathrm{~m} / \mathrm{s}$. After impact, the test film was evidently separated from the substrate, as shown in Fig. 2(b). The asymmetry of the separated configuration may be partly due to the inclined incidence of the bullet.

\section{Theoretical description of the method}

Here, the stress induced by the impact was analyzed with impact dynamics [8]. The acoustic impedances of the materials are denoted by $\rho_{i} c_{i}$, where $\rho_{i}$ is the density of the material and $c_{i}$ is the longitudinal elastic wave velocity, with the suffix $i=0,1,2$ and 3 . The suffix ' 0 ' represents the material of the bullet body, suffix ' 1 ' represents the coating on the bullet body, suffix ' 2 ' represents the substrate 


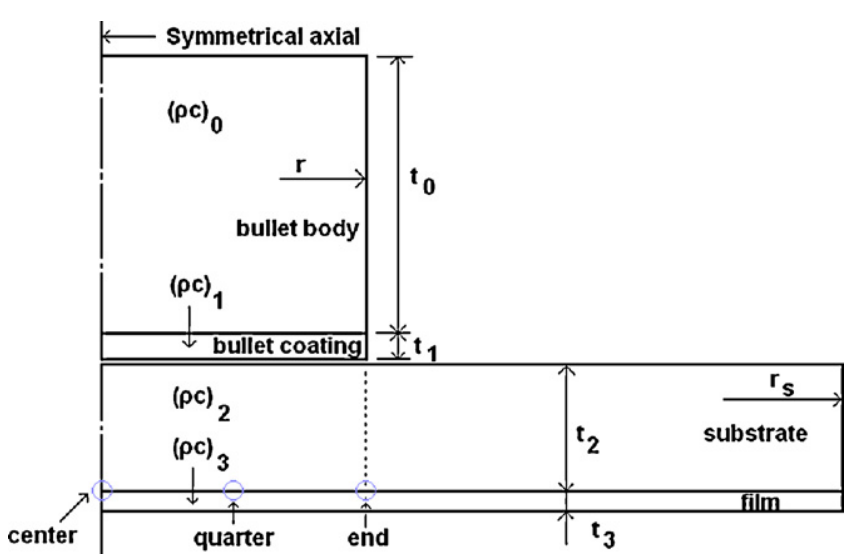

Fig. 1. Sketch of the impact method.

Table 1

Materials and mechanical parameters for the experiment.

\begin{tabular}{lllll}
\hline & Bullet body & Bullet coating & Substrate & Film \\
\hline Material & Plastic & Nickel & Steel & Nickel \\
Radius $(\mathrm{mm})$ & 12.7 & 12.7 & 100 & 100 \\
Thickness $(\mathrm{mm})$ & 45 (length) & 0.02 & 5 & 0.02 \\
Density $(\rho)\left(\mathrm{kg} \mathrm{m}^{-3}\right)$ & 1050 & 8800 & 7850 & 8800 \\
Elastic modulus $(E)(\mathrm{GPa})$ & 1.2 & 210 & 200 & 210 \\
Poisson's ratio $(v)$ & 0.4 & 0.31 & 0.29 & 0.31 \\
\hline
\end{tabular}

of the specimen under test, and suffix ' 3 ' represents the film of the specimen. The bullet with initial velocity $v_{0}$ is assumed to impact on the substrate of the specimen.

Under the impact, the stress arising within the bullet coating is

$\left.\sigma_{c}\right|_{\tau=0}=-\rho_{1} c_{1}\left(v_{0}-\left.v\right|_{\tau=0}\right)$

where

$\left.v\right|_{\tau=0}=v_{0}+\frac{\left.\sigma_{c}\right|_{\tau=0}}{\rho_{1} c_{1}}$

is the transient velocity of the particles at the contact interface of the bullet coating and the substrate of the specimen, while the stress arising in the substrate of the specimen is

$\left.\sigma_{s}\right|_{\tau=0}=-\left.\rho_{2} c_{2} v\right|_{\tau=0}=-\rho_{2} c_{2}\left(v_{0}+\frac{\left.\sigma_{c}\right|_{\tau=0}}{\rho_{1} c_{1}}\right)$

Upon contact, the principle of action and reaction result in

$\left.\sigma_{c}\right|_{\tau=0}=\left.\sigma_{s}\right|_{\tau=0}=\left.\sigma\right|_{\tau=0}$

Then, there holds

$\left.\sigma\right|_{\tau=0}=-v_{0} \frac{\rho_{1} c_{1} \rho_{2} c_{2}}{\rho_{1} c_{1}+\rho_{2} c_{2}}$

$$
\left.v\right|_{\tau=0}=v_{0} \frac{\rho_{1} c_{1}}{\rho_{1} c_{1}+\rho_{2} c_{2}}
$$

Thus, the velocity of the particles within the bullet coating relative to the interface between bullet coating and bullet body is

$v_{c p}=\left.v\right|_{\tau=0}-v_{0}=-v_{0} \frac{\rho_{2} c_{2}}{\rho_{1} c_{1}+\rho_{2} c_{2}}$

After the impact pulse has impinged at the interface between the bullet coating and the bullet body, the pulse will be partly reflected and transmitted. The reflected pulse is given by

$\sigma_{R}=\left.\sigma\right|_{\tau=0} \frac{\rho_{0} c_{0}-\rho_{1} c_{1}}{\rho_{0} c_{0}+\rho_{1} c_{1}}$

$v_{R}=-v_{c p} \frac{\rho_{0} c_{0}-\rho_{1} c_{1}}{\rho_{0} c_{0}+\rho_{1} c_{1}}$

Therefore, after the reflected pulse has propagated through the bullet coating, the velocity and the stress of the bullet coating obey

$\left.v_{c}\right|_{\tau=2 t_{1} / c_{1}}=v_{R}+\left.v\right|_{\tau=0}=\frac{1}{\rho_{1} c_{1}+\rho_{2} c_{2}}\left[\frac{\rho_{0} c_{0}-\rho_{1} c_{1}}{\rho_{0} c_{0}+\rho_{1} c_{1}} \rho_{2} c_{2}+\rho_{1} c_{1}\right] v_{0}$

$\left.\sigma_{c}\right|_{\tau=0}=\sigma_{R}+\left.\sigma\right|_{\tau=0}=-\frac{2 \rho_{0} c_{0}}{\rho_{0} c_{0}+\rho_{1} c_{1}} \frac{\rho_{1} c_{1} \rho_{2} c_{2}}{\rho_{1} c_{1}+\rho_{2} c_{2}} v_{0}$

If $\rho_{0} c_{0}-\rho_{1} c_{1} / \rho_{0} c_{0}+\rho_{1} c_{1} \rho_{2} c_{2}+\rho_{1} c_{1} \leq 0$, then $\left.v_{c}\right|_{\tau=2 t_{1} / c_{1}} \leq 0$, which indicates that the bullet coating will spring backwards or cease, and the unloading wave $\left(\sigma=0, v_{c p}=0\right)$ will be imported into the substrate. The stress pulse propagating in the specimen is $\sigma=-v_{0}\left(\rho_{1} c_{1} \rho_{2} c_{2} /\left(\rho_{1} c_{1}+\rho_{2} c_{2}\right)\right)$, with the duration of $2 t_{1} / c_{1}$.

If $\rho_{0} c_{0}-\rho_{1} c_{1} / \rho_{0} c_{0}+\rho_{1} c_{1} \rho_{2} c_{2}+\rho_{1} c_{1}>0$, then $\left.v_{c}\right|_{\tau=2 t_{1} / c_{1}}>0$. Before the second round of impact is sensed, the partial unloading wave will be imported into the substrate and it can be deduced similarly that the first main stress pulse $\sigma$ is $-v_{0}\left(\rho_{1} c_{1} \rho_{2} c_{2} / \rho_{1} c_{1}+\right.$ $\rho_{2} c_{2}$ ), with a tail of magnitude of

$\frac{4 \rho_{0} c_{0} \rho_{2} c_{2}}{\left(\rho_{0} c_{0}+\rho_{1} c_{1}\right)\left(\rho_{1} c_{1}+\rho_{2} c_{2}\right)}\left(-v_{0} \frac{\rho_{1} c_{1} \rho_{2} c_{2}}{\rho_{1} c_{1}+\rho_{2} c_{2}}\right)$

After impinging at the interface between the substrate and the film, the stress pulse will be partly reflected and transmitted again.

$\sigma_{r 1}=\frac{\rho_{3} c_{3}-\rho_{2} c_{2}}{\rho_{2} c_{2}+\rho_{3} c_{3}} \times \sigma$

$\sigma_{t}=\frac{2 \rho_{3} c_{3}}{\rho_{2} c_{2}+\rho_{3} c_{3}} \times \sigma$

The transmitted pulse will be reflected at the free surface of the film.

$\sigma_{r 2}=-\sigma_{t}$

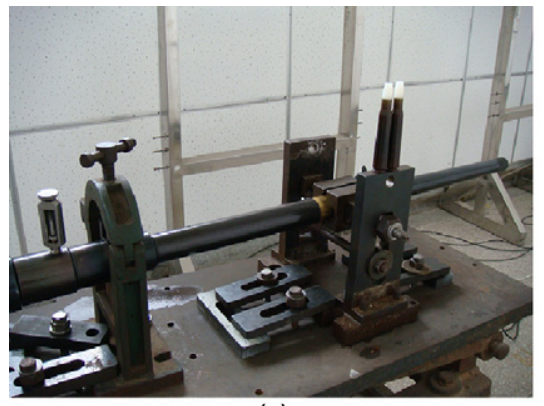

(a)

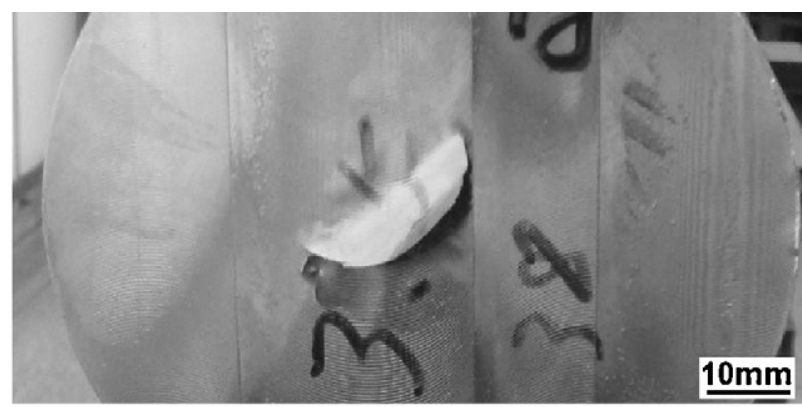

(b)

Fig. 2. Photos of the (a) experimental equipment and (b) the tested specimen. 
Table 2

Mechanical parameters used in FEA.

\begin{tabular}{lllll}
\hline & Bullet body & Bullet coating & Substrate & Film \\
\hline Density $(\rho)\left(\mathrm{kg} \mathrm{m}^{-3}\right)$ & 1050 & Variables & 7850 & 7190 \\
Elastic modulus $(E)(\mathrm{GPa})$ & 3.89 & Variables & 209 & 242 \\
Poisson's ratio $(v)$ & 0.35 & 0.3 & 0.3 & 0.15
\end{tabular}

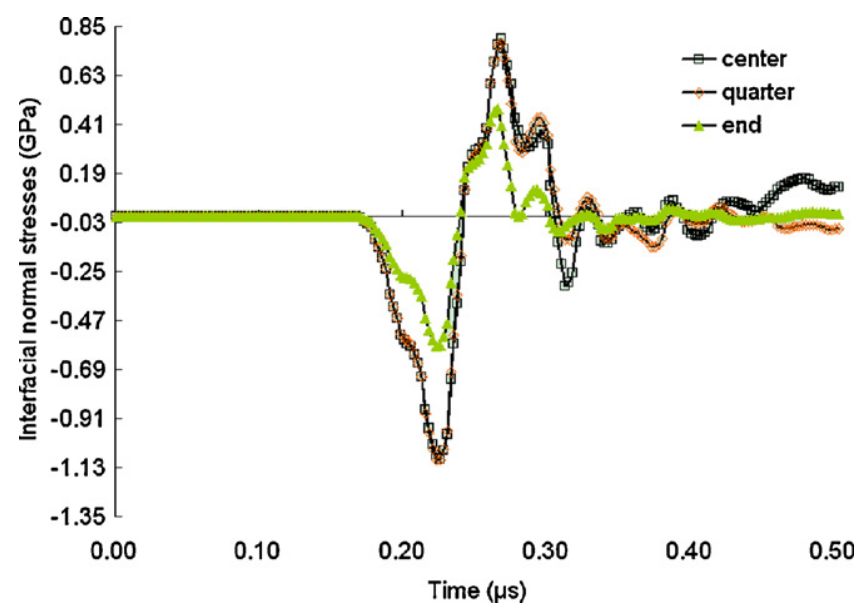

Fig. 3. Fluctuation of the interfacial normal stresses.

Herein, if the relationship $t_{2} \geq c_{3} \times 2 t_{1} / c_{1}$ is satisfied [4], then if $\rho_{0} c_{0}-\rho_{1} c_{1} / \rho_{0} c_{0}+\rho_{1} c_{1} \rho_{2} c_{2}+\rho_{1} c_{1} \leq 0$, then the stress should satisfy

$\sigma_{r 2}=v_{0} \rho_{1} c_{1} \frac{\rho_{2} c_{2}}{\rho_{1} c_{1}+\rho_{2} c_{2}} \frac{2 \rho_{3} c_{3}}{\rho_{2} c_{2}+\rho_{3} c_{3}}$

If $\rho_{0} c_{0}-\rho_{1} c_{1} / \rho_{0} c_{0}+\rho_{1} c_{1} \rho_{2} c_{2}+\rho_{1} c_{1}>0$, then the stress pulse is $\sigma_{r 2}$ with the tail, which can be obtained by combining (12) and (14). Moreover, the peak value of $\sigma_{r 2}$ should be

$v_{0} \rho_{1} c_{1} \frac{2 \rho_{2} c_{2} \rho_{3} c_{3}}{\left(\rho_{1} c_{1}+\rho_{2} c_{2}\right)\left(\rho_{2} c_{2}+\rho_{3} c_{3}\right)}\left(1-\frac{4 \rho_{0} c_{0} \rho_{2} c_{2}}{\left(\rho_{0} c_{0}+\rho_{1} c_{1}\right)\left(\rho_{1} c_{1}+\rho_{2} c_{2}\right)}\right)$

The results indicate that once the materials of the specimen are known, the peak value of $\sigma_{r 2}$ will increase with the increase of $\rho_{1} c_{1}$, which is the acoustic impedance of the bullet coating. Moreover, when $\rho_{0} c_{0}-\rho_{1} c_{1} / \rho_{0} c_{0}+\rho_{1} c_{1} \rho_{2} c_{2}+\rho_{1} c_{1}>0$, the peak value of $\sigma_{r 2}$ will increase with the decrease of the acoustic impedance of the bullet body. As an extreme case, maximum $\sigma_{r 2}$ will be attained under the assumption that the acoustic impedance of the bullet

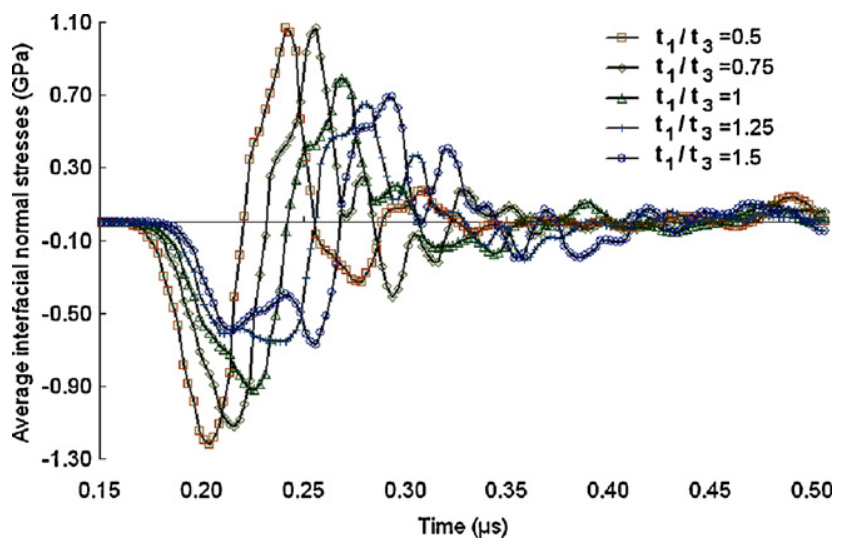

Fig. 4. Interfacial normal stresses for the case of $\left[(\rho E)_{1} /(\rho E)_{2}\right]^{0.5}=0.5$.

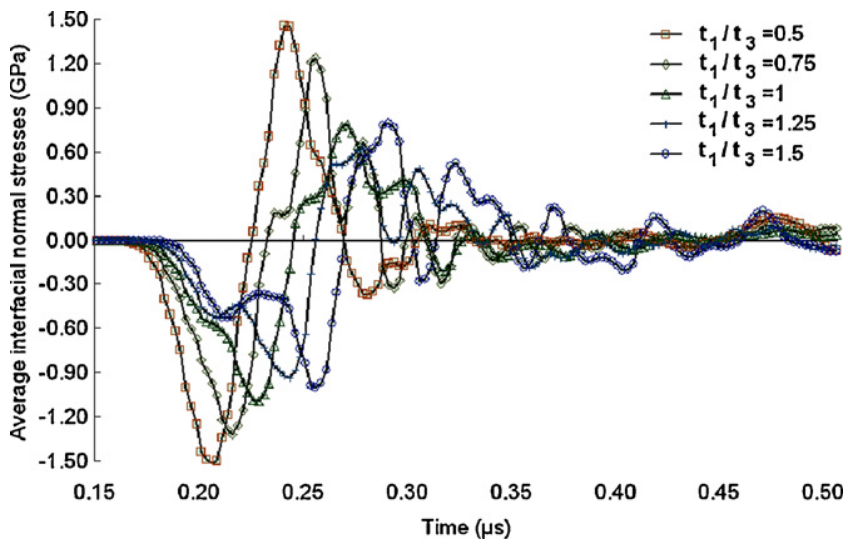

Fig. 5. Interfacial normal stresses for the case of $\left[(\rho E)_{1} /(\rho E)_{2}\right]^{0.5}=1$.

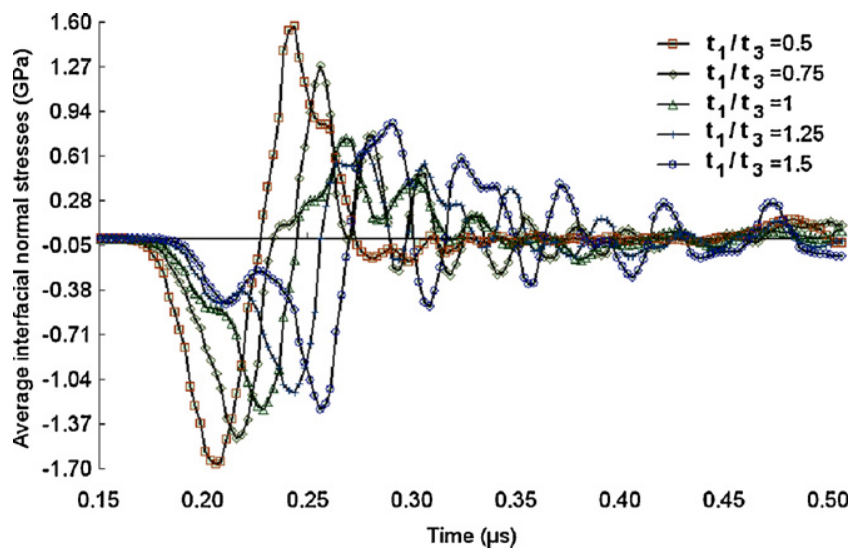

Fig. 6. Interfacial normal stresses for the case of $\left[(\rho E)_{1} /(\rho E)_{2}\right]^{0.5}=1.5$.

body is zero, which means that only the bullet coating impacts the specimen rather than the bullet.

\section{Parametric analysis of the process}

With finite element analysis, for the materials of mechanical parameters as shown in Table 2 with $(\rho E)_{1} /(\rho E)_{2}=1, t_{1} / t_{3}=1$ and $t_{2}=1.0 \mathrm{~mm}$, the interface stresses can be calculated. As shown in Fig. 3, the three curves represent the interface normal stresses of the nodes denoted in Fig. 1, in which 'center', 'quarter' and 'end' correspond to the loci of $x=0, x=r / 2$ and $x=r$. The results show

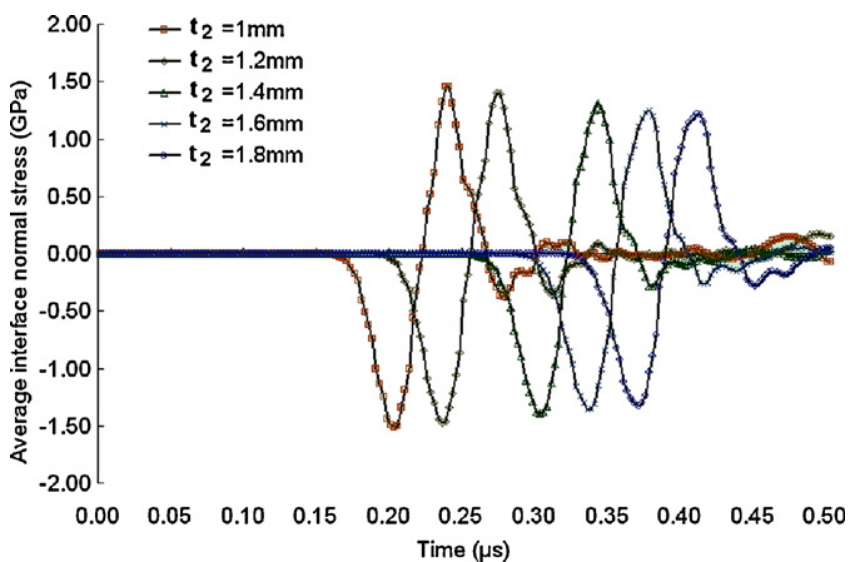

Fig. 7. Interfacial normal stresses for the cases with different values of $t_{2}$. (a) $t_{2}=2 \mathrm{~mm}$, (b) $t_{2}=4 \mathrm{~mm}$, (c) $t_{2}=6 \mathrm{~mm}$ and (d) $t_{2}=8 \mathrm{~mm}$ 


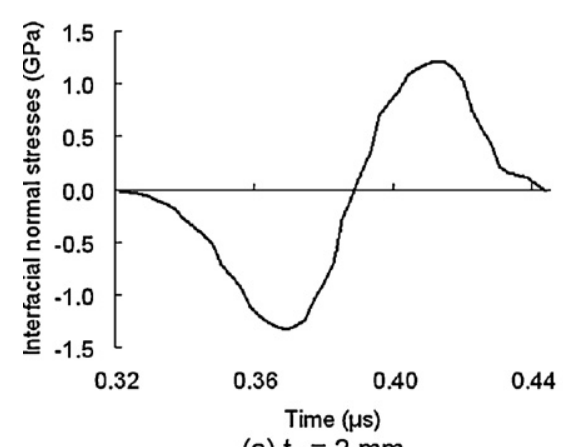

(a) $t_{2}=2 \mathrm{~mm}$

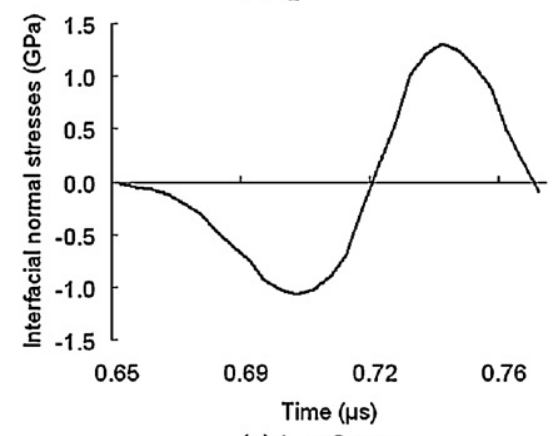

(c) $\mathrm{t}_{2}=6 \mathrm{~mm}$

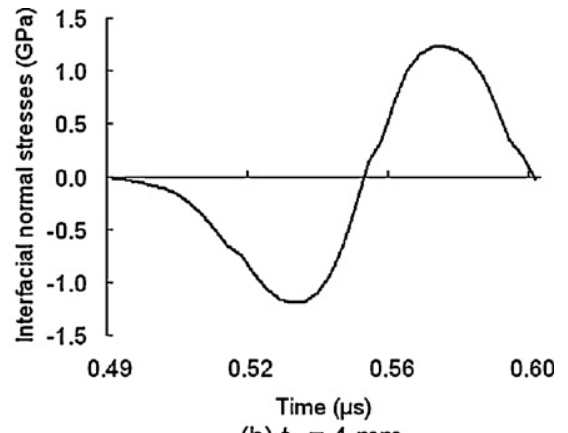

(b) $\mathrm{t}_{2}=4 \mathrm{~mm}$

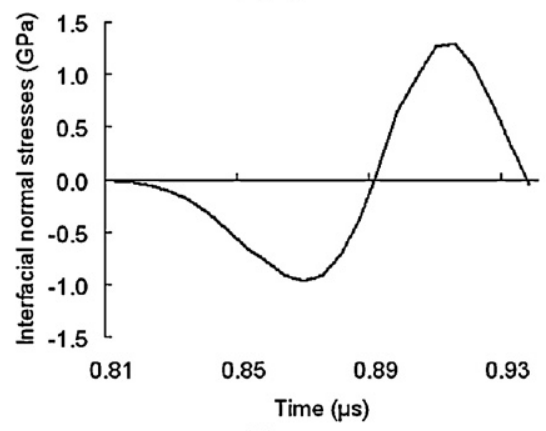

(d) $t_{2}=8 \mathrm{~mm}$

Fig. 8. Magnified profile of interfacial normal stresses for the cases with different values of $t_{2}$.

that the interface normal stresses are uniform, particularly when $x \leq r / 2$.

Thereafter, the effects of the parameters $(\rho E)_{1} /(\rho E)_{2}, t_{1} / t_{3}$ and $t_{2}$ were investigated. With the uniformity of the interface normal stresses being considered, the average value within the range when $x \leq r / 2$ was used for comparison.

Cases when $\left[(\rho E)_{1} /(\rho E)_{2}\right]^{0.5}=0.5,1$ and 1.5 were investigated, where $t_{1} / t_{3}=0.5,0.75,1,1.25$ or 1.5 for each case, respectively. Cases when $t_{2}=1,1.2,1.4,1.6,1.8,2,4,6$ and $8 \mathrm{~mm}$ were analyzed to grasp the transverse effect of the specimen.

The mechanical properties adopted in the calculation for bullet body, bullet coating, substrate and film are listed in Table 2 . The initial velocity of the bullet is $250 \mathrm{~m} / \mathrm{s}$.

Figs. 4-6 show the fluctuation of interface stresses for different ratios $t_{1} / t_{3}$ when $\left[(\rho E)_{1} /(\rho E)_{2}\right]^{0.5}=0.5,1$ and 1.5 , respectively. The results in Figs. 4-6 indicate that the interfacial normal stresses increase with increasing ratio $\left[(\rho E)_{1} /(\rho E)_{2}\right]^{0.5}$, while they decrease with increasing ratio $t_{1} / t_{3}$, suggesting that the acoustic impedance of the bullet coating should be great enough as well as its thickness low enough to obtain sufficiently high interface stresses.

Figs. 7 and 8 show the fluctuation of interface stresses for different $t_{2}$ values. The results indicate that the interface stresses decrease slightly with the thickness increase of the specimen substrate. In other words, the transverse effect of the specimen was small enough to be ignored when the thickness of the substrate was not more than $8 \mathrm{~mm}$, which is the maximum thickness considered in this study.

\section{Conclusions}

A preliminary experiment was performed, and the idea of the method of impact by a front-end-coated bullet to evaluate the inter- face adhesion of film and substrate was justified. After the substrate was impacted by a front-end-coated bullet, the test film was separated from the substrate.

The generalized form of interfacial normal stresses for the method with one-dimensional approximation is presented. The analytical solution shows that the interfacial normal stresses increase with the increase of the acoustic impedance of the bullet coating and with the decrease of the acoustic impedance the bullet body.

The results of numerical simulation further prove that the interfacial normal stresses decrease with increase of the thickness of the bullet coating. Besides, the transverse effect of the specimen becomes rather small as long as the thickness of the substrate is not more than $8 \mathrm{~mm}$ as used in this study.

\section{Acknowledgement}

This research was funded by the National Natural Science Foundation of China under grant NO. 50531060.

\section{References}

[1] L.B. Freund, S. Suresh, Thin Film Materials, Cambridge University Press, Cambridge, 2003, p. 239

[2] C.H. Hsueh, Appl. Phys. Lett. 81 (2002) 1615

[3] Robert Lacombe, Adhesion Measurement Methods: Theory and Practice, CRC Press, London, 2006, p. 7.

[4] Chen-Wu Wu, Zhi-Lin Wu, Kun Zhang, Guang-Nan Chen, A method of impact with the coated bullet to evaluate the adhesion between the film and substrate, J. Mech. Mater. Struct., in press http://pjm.math.berkeley.edu/wft/status. php?p_id=15106\&cr=2ad5656e1441b0c3.

[5] J.L. Vossen, ASTM STP 640 (1978) 122.

[6] V. Gupta, A.S. Argon, J.A. Cornie, D.M. Parks, Mater. Sci. Eng. A 126 (1990) 105.

[7] V. Gupta, J. Yuan, J. Appl. Phys. 74 (1993) 2397.

[8] L.L. Wang, Foundation of Stress Waves, 2nd ed., National Defense Industry Press, Beijing, China, 2005, pp. 29 and 47 (in Chinese). 